This article has been accepted for publication in Monthly Notices of the Royal Astronomical Society (C: 2018 The Authors Published by Oxford University Press on behalf of the Royal Astronomical Society. All rights reserved. 


\title{
Discovery of large-scale diffuse radio emission in low-mass galaxy cluster Abell 1931
}

\author{
M. Brüggen, ${ }^{1 \star}$ D. Rafferty, ${ }^{1}$ A. Bonafede, ${ }^{1,2}$ R. J. van Weeren,,${ }^{3,4}$ T. Shimwell, ${ }^{3}$ \\ H. Intema, ${ }^{3}$ H. Röttgering, ${ }^{3}$ G. Brunetti, ${ }^{2}$ G. Di Gennaro, ${ }^{3,4}$ F. Savini, ${ }^{1}$ A. Wilber, ${ }^{1}$ \\ S. O’Sullivan, ${ }^{1}$ T. A. Ensslin, ${ }^{5}$ F. De Gasperin ${ }^{1,3}$ and M. Hoeft ${ }^{6}$ \\ ${ }^{1}$ Hamburger Sternwarte, University of Hamburg, Gojenbergsweg 112, D-21029 Hamburg, Germany \\ ${ }^{2}$ Istituto di Radio Astronomia, INAF, Via Godetti 101, I-40121 Bologna, Italy \\ ${ }^{3}$ Leiden Observatory, Leiden University, PO Box 9513, NL-2300 RA Leiden, the Netherlands \\ ${ }^{4}$ Harvard-Smithsonian Center for Astrophysics, 60 Garden Street, Cambridge, MA 02138, USA \\ ${ }^{5}$ Max-Planck-Institut für Astrophysik, Karl-Schwarzschild-Str 1, Postfach 1317, D-85741 Garching, Germany \\ ${ }^{6}$ Thüringer Landessternwarte, Sternwarte 5, D-07778 Tautenburg, Germany
}

Accepted 2018 March 28. Received 2018 March 23; in original form 2018 March 2

\begin{abstract}
Extended, steep-spectrum radio synchrotron sources are pre-dominantly found in massive galaxy clusters as opposed to groups. LOFAR Two-Metre Sky Survey images have revealed a diffuse, ultra-steep-spectrum radio source in the low-mass cluster Abell 1931. The source has a fairly irregular morphology with the largest linear size of about $550 \mathrm{kpc}$. The source is only seen in LOFAR observations at $143 \mathrm{MHz}$ and Giant Metre Radio Telescope observations at $325 \mathrm{MHz}$. The spectral index of the total source between 143 and $325 \mathrm{MHz}$ is $\alpha_{143}^{325}=$ $-2.86 \pm 0.36$. The source remains invisible in Very Large Array $(1-2 \mathrm{GHz})$ observations as expected given the spectral index. Chandra X-ray observations of the cluster revealed a bolometric luminosity of $L_{X}=(1.65 \pm 0.39) \times 10^{43} \mathrm{erg} \mathrm{s}^{-1}$ and a temperature of $2.92_{-0.87}^{+1.89} \mathrm{keV}$ which implies a mass of around $\sim 10^{14} \mathrm{M}_{\odot}$. We conclude that the source is a remnant radio galaxy that has shut off around 200 Myr ago. The brightest cluster galaxy, a radio-loud elliptical galaxy, could be the source for this extinct source. Unlike remnant sources studied in the literature, our source has a steep spectrum at low radio frequencies. Studying such remnant radio galaxies at low radio frequencies is important for understanding the scarcity of such sources and their role in feedback processes.
\end{abstract}

Key words: acceleration of particles-intergalactic medium-radiation mechanisms: nonthermal - radio continuum: galaxies - X-rays: galaxies: clusters.

\section{INTRODUCTION}

Diffuse radio sources in galaxy clusters, radio relics and haloes, are signatures of dynamic cluster events. Radio relics are low surface brightness $\left(\simeq 10^{-6} \mathrm{Jy} \mathrm{arcsec}^{-2}\right.$ at $\left.1.4 \mathrm{GHz}\right)$, steep-spectrum ${ }^{1}$ $(\alpha<-1)$ sources that are typically located in the periphery of galaxy clusters. While it is known that non-thermal electrons are responsible for the observed synchrotron radiation, the mechanism of particle acceleration as well as the origins of the magnetic fields on these scales are poorly understood (Brunetti \& Jones 2014; Vazza et al. 2017).

\footnotetext{
*E-mail: mbrueggen@hs.uni-hamburg.de

${ }^{1} S(v) \propto v^{\alpha}$
}

Radio relics trace low-Mach number shocks $(\mathcal{M} \lesssim 3)$ generated by cluster-cluster merger events. A long-standing problem is how low-Mach number shocks can accelerate electrons so efficiently to explain the bright radio relics that are observed. Current theories of diffusive shock acceleration have difficulties in explaining the required efficiency of particle acceleration in the low-Mach number shocks as found in galaxy clusters (e.g. Kang \& Ryu 2011; Guo, Sironi \& Narayan 2014). In particular, it remains unclear what role pre-existing populations of relativistic electrons play. It has been suggested that these fossil electrons, with Lorentz factors of $~ 200$ and lifetimes of several Gyr, can be efficiently re-accelerated at shocks and are therefore able to create bright radio relics (Ensslin et al. 1998; Kempner et al. 2004; Kang \& Ryu 2015; Pinzke, Oh \& Pfrommer 2013, sometimes called 'gischt' relics).

Radio haloes are Mpc-sized, synchrotron sources that fill the central regions of galaxy clusters. They arise from the accelera- 
tion of particles by turbulence generated during cluster mergers. Hadronic models that involve the generation of secondary particles via proton-proton collisions in the intracluster medium (ICM; Dennison 1980) are believed to play a lesser role (Brunetti \& Jones 2014). However, hadronic models could be important for minihaloes (Pfrommer \& Enßlin 2004).

Most of the information on the statistical connection between cluster mass, dynamics, and radio haloes are obtained by surveys of X-ray luminous galaxy clusters that have been carried out with radio telescopes (Venturi et al. 2008; Kale et al. 2013, 2015). There is strong evidence that radio haloes and relics depend on the dynamical state of the clusters (e.g. Wen \& Han 2013; Cuciti et al. 2015).

In the turbulent re-acceleration model at the low-mass end, only very disturbed clusters host diffuse radio sources and the slope of the $P_{140 \mathrm{MHz}}-L_{\mathrm{X}}$ relation steepens at lower luminosities (Cassano et al. 2010). A subclass of radio haloes with ultra-steep spectra mostly lie slightly below the scaling relation, potentially filling in the region between the correlation and the upper limits (e.g. Brunetti et al. 2008; Wilber et al. 2018).

Another class of steep-spectrum radio sources that can be found in galaxy clusters are so-called radio phoenices and active galactic nucleus (AGN) relics, which are both related to radio galaxies. AGN relics are essentially extinct or dying radio galaxies. Their radio spectra are steep owing to the synchrotron and inverse Compton losses. Fossil radio plasma from past episodes of AGN activity can also be compressed by shock waves. The ensuing increase in the momentum of the relativistic electrons as well as the increase in the magnetic field strength can produce a radio phoenix (Enßlin \& Gopal-Krishna 2001; Enßlin \& Brüggen 2002). Again, these sources have steep and curved radio spectra. The distribution of fossil plasma throughout clusters is an important question since old populations of relativistic electrons are required for most acceleration models that explain radio relics and haloes. They also bear witness to past AGN activity and constitute a source of nonthermal pressure in the ICM. The study of past radio-loud AGN activity is important for quantifying of AGN feedback, which is key in preventing catastrophic cooling in the centres of galaxy clusters. Murgia et al. (2011) have studied remnant sources in galaxy clusters and suggest that the duration of the fading phase of remnant radio galaxies in clusters is longer than in the field. The reason may be that the expansion of the radio lobes in clusters has to work against a higher ambient pressure.

Based on the number density of radio galaxies, one would expect a large number of remnant radio sources. Yet, observations show that they are exceptionally rare (Giovannini et al. 1988; Parma et al. 2007). Recently, Brienza et al. (2016) found an AGN relic or remnant radio galaxy in LOFAR images at $150 \mathrm{MHz}$ that extends over $700 \mathrm{kpc}$ but is not associated with a galaxy cluster. Also its spectrum is not steep at low frequencies and only steepens at frequencies >1.4 GHz. In follow-up work, Brienza et al. (2017) selected from the LOFAR Lockman Hole field 23 candidates for remnant galaxies, all outside of galaxy clusters. Interestingly, many of these remnants do not have very steep spectra.

Savini et al. (2018) have discovered an extended radio galaxy embedded in steep diffuse emission in a galaxy group. The spectral index map is inconclusive as to whether this source is still active. Detections of remnant radio galaxies are surprisingly rare. Dwarakanath \& Kale (2009) have studied a sample of relic galaxies with the Giant Metre Radio Telescope (GMRT) and the VLA. They find that the sources that show no obvious cores and jets have steep spectra that suggest that the AGN activity has stopped 15-100 million years ago. Before that, these sources were up to 1000 times brighter than their current luminosities making them comparable to the brightest AGN in the local Universe.

Godfrey, Morganti \& Brienza (2017) find that fewer than 2 per cent of FRII radio galaxies with $S_{74 \mathrm{MHz}}>1.5 \mathrm{Jy}$ are ultrasteep-spectrum remnants. They define an ultra-steep spectrum as spectra with indices between $74 \mathrm{MHz}$ and $1.4 \mathrm{GHz}$ of $<-1.2$. Godfrey et al. (2017) have modelled the evolution of the remnants in order to match the low number of observed sources. They suggest that the remnants must have a rapid luminosity evolution and keep on expanding, adiabatically, after the core switches off. This was done by comparing mock samples with observations. In Godfrey et al. (2017) this was done for FRII sources and in Brienza et al. (2017) for FRI sources.

Since radio relics have steep radio spectra, they are best discovered at low radio frequencies. However, until now, only shallow low-frequency radio surveys have been carried out (i.e. the $150 \mathrm{MHz}$ TGSS ADR survey, the $325 \mathrm{MHz}$ WENSS survey, and the $74 \mathrm{MHz}$ VLSSr survey, Rengelink et al. 1997; Lane et al. 2014; Intema et al. 2017). The LOFAR Two-Metre Sky Survey (LoTSS; Shimwell et al. 2017) is a new low-frequency survey aiming to map the entire northern sky. When completed, it will be two orders of magnitude deeper (in point-source sensitivity) and one order of magnitude higher in resolution than any current very large radio survey. The first 600 pointings ( 20 per cent of the total) of this survey have recently been observed, covering the 120-168 MHz band.

Here, we investigate the nature of the diffuse radio source in the galaxy cluster Abell 1931 using data from LOFAR (120-168 MHz), the GMRT (325 MHz), the Very Large Array (VLA) (1-2 GHz), and the Chandra X-ray observatory $(0.5-7 \mathrm{keV})$. We chose to study the galaxy cluster Abell 1931 because it was believed to have a relatively low mass (no ROSAT detection) and showed large-scale diffuse emission in LOFAR images that was not seen in other radio surveys. Steep and diffuse radio emission in low-mass clusters can yield new insights into the nature of the acceleration mechanism, the role of fossil electrons, and the origin of intracluster magnetic fields Vazza et al. (2017).

In this paper we adopt a flat, $\Lambda \mathrm{CDM}$ cosmology with matter density $\Omega_{\mathrm{M}}=0.3$ and Hubble constant $H_{0}=67.8 \mathrm{~km} \mathrm{~s}^{-1} \mathrm{M} \mathrm{pc}^{-1}$ (Planck Collaboration XIII 2016). Given the redshift of Abell 1931 ( $z=0.178$ ), the luminosity distance is $D_{\mathrm{L}}=889 \mathrm{Mpc}$ and $1 \operatorname{arcsec}$ corresponds to $3.109 \mathrm{kpc}$. All our images are in the J2000 coordinate system.

\section{A NEW ULTRA-STEEP-SPECTRUM SOURCE IN ABELL 1931}

\subsection{LOFAR observations}

LOFAR is a digital, low-frequency radio interferometer that extends over large parts of Northern Europe and has its core in the Northern parts of the Netherlands (van Haarlem et al. 2013). The observation shown here was part of the LoTSS (Shimwell et al. 2017) and was conducted with the high-band antennas (HBA) over a frequency range of 120-168 MHz. For a summary of all radio observations, see Table 1 .

After a complete direction-independent calibration of the data, we apply Facet Calibration. Facet Calibration is a direction-dependent calibration method for LOFAR in which the full field is tessellated into multiple facets (van Weeren et al. 2016a). The full field-of-view (FOV) that is approximately $3 \mathrm{deg}$ across is thus split into up to 50 facets. Each facet has its own calibrator source, chosen to have at least $0.3 \mathrm{Jy}$ in flux density. Facet Calibration is applied using the 
Table 1. List of radio observations on A1931.

\begin{tabular}{|c|c|c|c|c|}
\hline & LOFAR & GMRT & VLA B & VLA C \\
\hline Observation date & 2015 March 16 & 2016 June 6 & 2017 December 18 & 2017 May 29 \\
\hline Bandwidth & $48 \mathrm{MHz}$ & $33 \mathrm{MHz}$ & $1 \mathrm{GHz}$ & $1 \mathrm{GHz}$ \\
\hline On-source time & $8 \mathrm{~h}$ & $6 \mathrm{~h}$ & $7 \mathrm{~h}$ & $5 \mathrm{~h}$ \\
\hline Noise & $180 \mu \mathrm{Jy}$ beam $^{-1}$ & $55 \mu \mathrm{Jy}$ beam $^{-1}$ & $18 \mu \mathrm{Jy}$ beam $^{-1}$ & $18 \mu \mathrm{Jy}$ beam $^{-1}$ \\
\hline
\end{tabular}

software package FACTOR ${ }^{2}$ which removes time and position-varying beam errors and ionospheric effects, which can be severe at these frequencies. It was ensured that the emission from the calibrator was deconvolved until the residual clean images were noise like. For the pointing P218+45 (containing Abell 1931), the resulting image has a noise level of $\sim 180 \mu \mathrm{Jy}$ beam $^{-1}$ and an angular resolution of about 5 arcsec. The noise was determined from a circular region free of sources.

From the image, an extended, steep-spectrum source was found near the low-mass cluster Abell 1931. This source is faint and no radio emission is detected in the NVSS and VLSS surveys. The source has a fairly irregular morphology with the largest linear size of about $550 \mathrm{kpc}$.

From the centre of the source (at approximately $14^{\mathrm{h}} 32^{\mathrm{m}} 11^{\mathrm{s}}$, $+44^{\mathrm{d}} 15.5^{\mathrm{m}}$ ), there appear to be two arms: one extending to the south-east with a length of about 1 arcmin and a width of 0.25 arcmin and the other extending to the North with a length of about $1 \mathrm{arcmin}$ and a width of $0.5 \mathrm{arcmin}$. There is no evidence for edge brightening or other distinct features such as jets or hotspots.

The diffuse emission appears to overlap with an overdensity of galaxies as seen in the Sloan Digital Sky Survey (SDSS). From the SDSS image, the source appears to be located in a very elongated cluster that is extended in the NE-SW direction, see Fig. 1. The morphology suggests that this cluster is very likely undergoing a merger with the major axis of the source being perpendicular to the merger axis. The centre of the cluster is quoted at $14^{\mathrm{h}} 31^{\mathrm{m}} 59^{\mathrm{s}}$, $+44^{\mathrm{d}} 15.8^{\mathrm{m}}$ on NED, ${ }^{3}$ but the SDSS galaxy distribution suggests that the cluster extends further to the East. A bright, red elliptical galaxy (SDSS J143212.84+441620.4), possibly the brightest cluster galaxy (BCG), has a radio counter-part and appears to be active. It has a spectroscopic redshift of $z=0.180$, an $r$-band magnitude of 16.94 and lies at coordinates $14^{\mathrm{h}} 32^{\mathrm{m}} 13^{\mathrm{s}},+44^{\mathrm{d}} 16.26^{\mathrm{m}}$.

The cluster redshift has been quoted as 0.170 (photometric redshift only, Lopes et al. 2004). Three SDSS cluster galaxies (based on their colours) with spectroscopic redshift are available in the vicinity that yield a redshift of $z=0.178$, consistent with the photometric redshift.

It is quite plausible that the BCG could be one of the suppliers of the relativistic electrons that produce the synchrotron emission in the diffuse source. The connection of AGN with diffuse radio sources is the topic of a recent series of papers (e.g. Bonafede et al. 2014; de Gasperin et al. 2014, 2017; van Weeren et al. 2017; Wilber et al. 2018).

From the primary-beam-corrected image, we measure a total flux density for the source within the $3 \sigma$ contours at $143 \mathrm{MHz}$ of $S_{143 \mathrm{MHz}}=39.7 \pm 8.0 \mathrm{mJy}$ [this is after correcting for the global flux

\footnotetext{
${ }^{2} \mathrm{http}: / /$ www.astron.nl/citt/facet-doc/

${ }^{3}$ https://ned.ipac.caltech.edu
}

offset relative to fluxes from the TIFR GMRT Sky Survey (TGSS) (Intema et al. 2017)]. The error includes a statistical error, estimated for the image noise in a nearby source-free region, and a flux scale uncertainty of 20 per cent.

For a redshift of $z=0.178$, the luminosity distance is 888.9 $\mathrm{Mpc}$, yielding a specific luminosity (including the $k$-correction as in Cassano et al. (2013)) of $P_{143 \mathrm{MHz}}=2.78 \pm 0.56 \times 10^{24} \mathrm{~W} \mathrm{~Hz}^{-1}$.

\subsection{GMRT observations}

Subsequently, we observed the cluster A1931 with the GMRT at $325 \mathrm{MHz}$ under Director's discretionary time. It was observed on 2016 June 15 for $6 \mathrm{~h}$ with an integration time of $8 \mathrm{~s}$. The data were processed using the SPAM pipeline (see Intema et al. 2017, for details).

Both bandpass and absolute flux scale were calibrated using the source 3C286 as primary flux calibrator and applying the Scaife \& Heald (2012) flux scale. We performed an initial phase-only calibration using a sky model generated from the surveys VLSSr, WENSS, and the NVSS. SPAM uses AIPS tasks (Greisen 2003) to perform (faceted) wide-field imaging of the full field of view, followed by a series of self-calibration, wide-field imaging, and additional flagging of poor data where needed. Then we used the brightest sources in the primary beam to conduct a direction-dependent calibration. Finally, SPAM fits ionospheric models to mitigate phase errors. The resulting image is shown in Fig. 2 and was made using slightly uniform weighting (ROBUST $=-1$ ). Note that the AIPS ROBUST parameter is a bit less strong than the CASA one.

We measure a total flux density for the diffuse emission from the GMRT image of $S_{325 \mathrm{MHz}}=3.85 \pm 0.82 \mathrm{mJy}$ (using the same region used for the LOFAR measurement, defined from the $3 \sigma$ contours at $143 \mathrm{MHz}$ ), where we have adopted a flux scale uncertainty of 20 percent. The corresponding power is $P_{325 \mathrm{MHz}}=2.7 \pm 0.58 \times 10^{23} \mathrm{~W} \mathrm{~Hz}$. The spectral index of the total source between 143 and $325 \mathrm{MHz}$ is therefore $\alpha_{143}^{325}=-2.86 \pm 0.36$.

We note that, although the LOFAR and GMRT images are not matched precisely in terms of weighting and UV-coverage, both images should be equally sensitive to emission on the scales of the diffuse emission studied here $\left(\sim 2^{\prime}\right)$.

\subsection{VLA observations}

Abell 1931 was observed on 2017 May 29 and 2017 December 18 with the Jansky VLA in C- and B-configurations, respectively. The observations were carried out in the $1-2 \mathrm{GHz} L$ band with a total on-source time of about 5 and $7 \mathrm{~h}$, respectively. We used the default frequency set-up which comprises two $512 \mathrm{MHz}$ IF pairs (each comprising eight contiguous subbands of $64 \mathrm{MHz}$ ) to cover the entire $1-2 \mathrm{GHz}$ of the $L$-band receiver. The data reduction was per- 


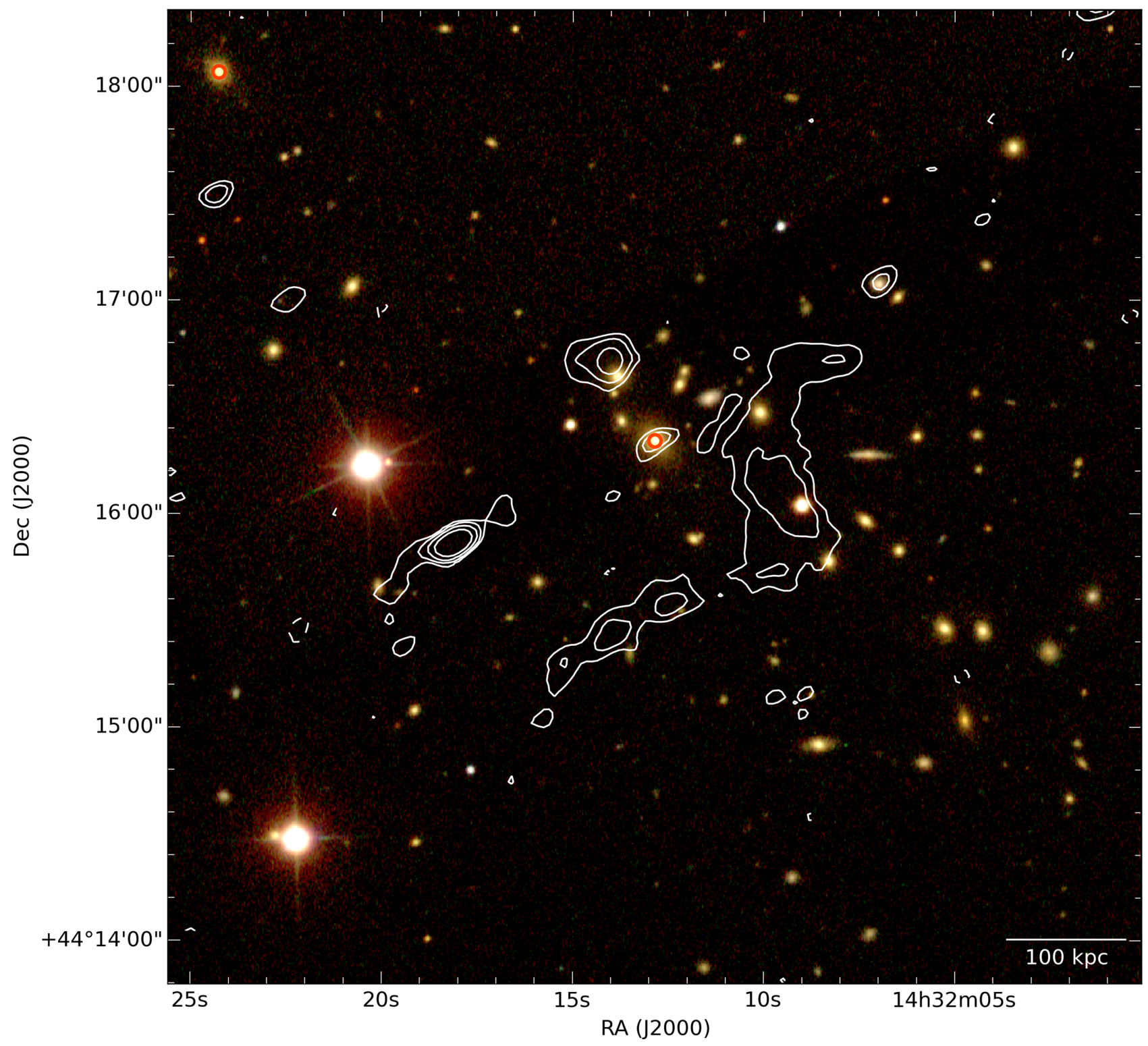

Figure 1. Overlay of SDSS $i-, r$-, $g$-band image with LOFAR HBA 120-168 MHz image of Abell 1931 with a noise of about $180 \mu \mathrm{Jy}^{\text {beam }}{ }^{-1}$. Contour levels are drawn at $[ \pm 3,6,12,24] \sigma\left(\sigma=180 \mu \mathrm{Jy}\right.$ beam $\left.^{-1}\right)$. Galaxies with known redshifts are marked by red rings. The galaxy distribution seems to be elongated along a NE-SW axis, although many galaxies do not have confirmed redshifts.

formed in CASA (v5.1; McMullin et al. 2007), following the scheme described in van Weeren et al. (2016b). We briefly summarize the main steps: First, the data were Hanning smoothed and corrected for elevation dependent gains and antenna offset positions. Radio frequency interference was removed with the 'tfcrop' mode in flagdata and in the final round with the aoflagger tool (Offringa et al. 2010). We calibrated the antenna delays, bandpass, crosshand delays, and polarization leakage and angles using the primary calibrators 3C147 and 3C286. Gain solutions were then obtained for the secondary calibrator source and the relevant calibration tables were applied to the target field. Self-calibration was performed to refine the amplitude and phase calibration on the target. All imaging was done with W-projection (Cornwell, Golap \& Bhatnagar 2008, 2005) and multi-scale, multi-frequency synthesis (MS-MFS) (Rau \& Cornwell 2011) with three Taylor terms (nterms=3). For all self-calibration steps, we employed Briggs weighting (Briggs
1995), with a robust factor of 0.0. Clean masks were also made with the pybdsf package (Mohan \& Rafferty 2015). After selfcalibration, the data from the two array configurations were combined and a final gain calibration step was performed with a long solution interval $(10 \mathrm{~min})$ to align the data sets. The final images were corrected for the primary beam attenuation.

The resulting image is shown in Fig. 2. For regular sources with spectral indices of $\sim-0.5$, the VLA image goes significantly deeper than LOFAR the LOFAR image. Still, the diffuse LOFAR source remains undetected. We can place an upper limit on the value of the spectral index of the diffuse emission between 143 and $1500 \mathrm{MHz}$ by assuming the emission would fill the same region in the VLA image with a uniform surface brightness. Under these assumptions, any such emission must have a total flux of $S_{1-2 \mathrm{GHz}}<0.52 \mathrm{mJy}$ to remain undetected in the VLA image, implying a spectral index between $143 \mathrm{MHz}$ and $1.5 \mathrm{GHz}$ of $\alpha_{143}^{1500}<-1.9$. With a redshift 

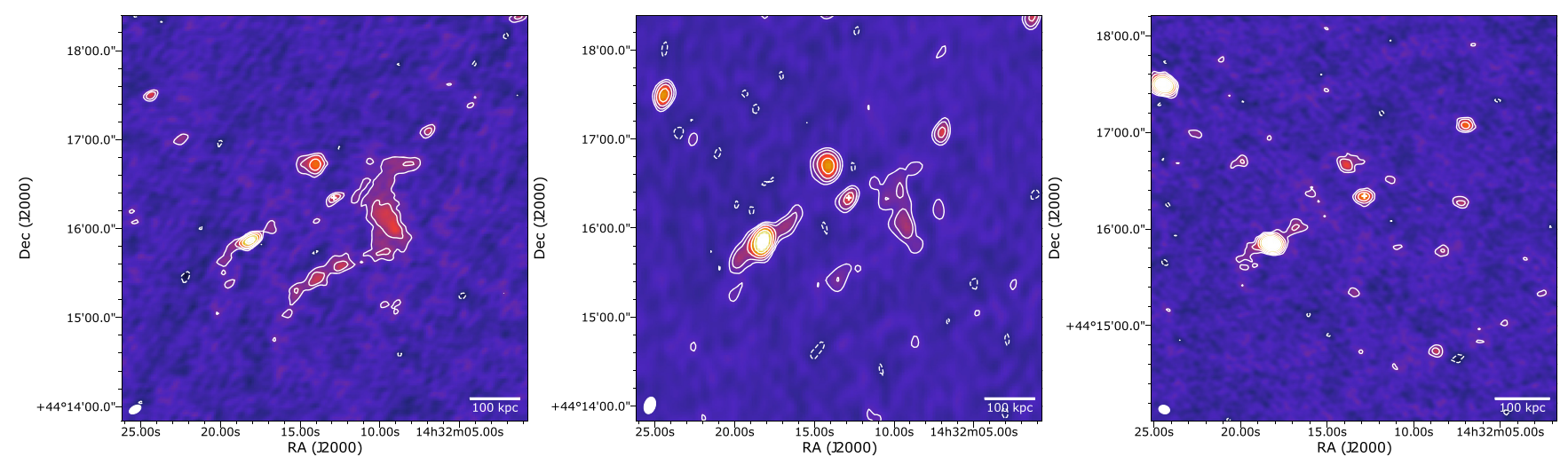

Figure 2. Left: LOFAR HBA $120-168 \mathrm{MHz}$ image. Contour levels are drawn at $[ \pm 3,6,12,24] \times \sigma\left(\sigma=180 \mu \mathrm{Jy}\right.$ beam $\left.^{-1}\right)$ and the restoring beam of $5.0 \times 8.7 \mathrm{arcsec}$ is shown by the ellipse in the lower left corner. Middle: GMRT $325 \mathrm{MHz}$ image. Contour levels are drawn at $[ \pm 3,6,12,24] \times \sigma(\sigma=55$ $\mu \mathrm{Jy}$ beam $\left.^{-1}\right)$; the restoring beam is $7.5 \times 11.6$ arcsec. Right: VLA $1-2 \mathrm{GHz} \mathrm{B}+\mathrm{C}$ array image. Contour levels are drawn at $[ \pm 3,6,12,24] \times \sigma(\sigma=18$ $\mu \mathrm{Jy}$ beam $\left.^{-1}\right)$; the restoring beam is $5.2 \times 7.0$ arcsec. The cross marks the location of the BCG. No emission from the extended LOFAR source is detected in the VLA image.

of $z=0.178$ this translates into an upper limit on the power of $P_{1.5 \mathrm{GHz}}<4.9 \times 10^{22} \mathrm{~W} \mathrm{~Hz}^{-1}$.

\section{X-RAY OBSERVATIONS OF ABELL 1931}

After the discovery of the diffuse emission with LOFAR, we proposed for X-ray observations to determine the mass of the cluster. Abell 1931 was observed with the Chandra X-ray observatory (ObsId: 19569, VFAINT mode) in Cycle 18 on 2017 September 28 with a total exposure time of $40.5 \mathrm{ks}$, using the ACIS-I detector. The data were reprocessed with CIAO $4.9^{4}$ using CALDB $4.7 .6^{5}$ and were corrected for known time-dependent gain and charge transfer inefficiency problems. Blank-sky background files were used for background subtraction. ${ }^{6}$ The events files were filtered for flares using the CIAO script $l c_{-}$clean to match the filtering used during the construction of the background files. A total of $5.7 \mathrm{ks}$ was removed during filtering, resulting in a final exposure time of $34.8 \mathrm{ks}$. The background files were normalized to the count rate of the source image in the $10-12 \mathrm{keV}$ band (after filtering). The derived normalization ratio (in the sense of background/source) was 1.12. Lastly, point sources detected using the CIAO tool wavdetect were removed. The X-ray map is shown in Fig. 3. In accordance with the appearance of the galaxy distribution the ICM is elongated in the NE-SW direction.

For the spectral analysis, spectra were extracted using the CIAO script SPECEXTRACT. For each spectrum, weighted responses were made, and a background spectrum was extracted in the same region of the CCD from the associated blank-sky background file. For the spectral fitting, XSPEC (Arnaud 1996) version 12.7.1 was used. $\mathrm{X}$-ray spectra were extracted in a circular region centred on the approximate centroid of the cluster emission (at $\alpha=14: 32: 14.7$, $\delta=+44: 16: 22.2$ ) with radius of 136 arcsec containing $\approx 400$ background-subtracted counts. Gas temperatures were found by deprojecting these spectra using the PROJCT model in XSPEC with a single-temperature plasma component (MEKAL) absorbed by foreground absorption component (WABS), between the energies of 0.5 and $7.0 \mathrm{keV}$. In this fitting, the redshift was fixed to

\footnotetext{
${ }^{4}$ See cxc.harvard.edu/ciao/index.html

${ }^{5}$ See cxc.harvard.edu/caldb/index.html

${ }^{6}$ See http://asc.harvard.edu/contrib/maxim/acisbg/
}

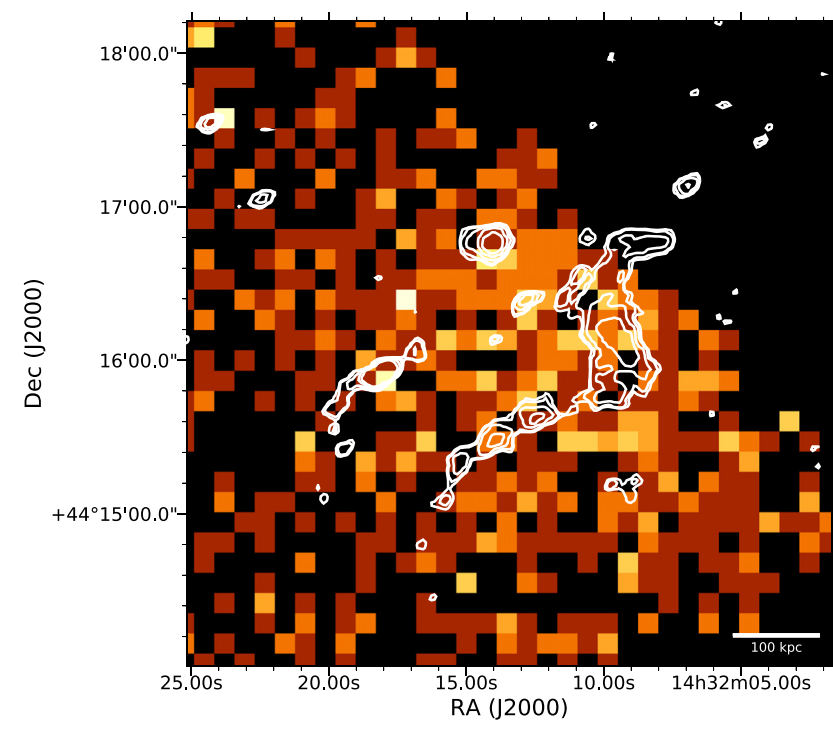

Figure 3. Chandra map with LOFAR contours overlaid. The LOFAR contours start at $3 \sigma\left(0.56 \mathrm{mJy}^{-1}\right.$ beam $\left.^{-1}\right)$ and increase by a factor of 2 . The image $(0.5-2 \mathrm{keV}$, exposure-corrected) was binned such that each pixel is $16 \times 16$ original pixels in order to make the emission more visible. The cross marks the location of the BCG.

$z=0.178$, and the foreground hydrogen column density was fixed to $N_{\mathrm{H}}=1.55 \times 10^{20} \mathrm{~cm}^{-2}$, the weighted-average Galactic value from Dickey \& Lockman (1990). The metallicity of the MEKAL component was fixed to 0.4 solar, using Anders \& Grevesse (1989) abundances.

The total X-ray luminosity within a radius of $450 \mathrm{kpc}$ is $L_{X}=(1.65 \pm 0.39) \times 10^{43} \mathrm{erg} \mathrm{s}^{-1}$. Using the $M-L_{X}$ relation by Reichert et al. $(2011), M_{500}=1.39 \times 10^{44}\left(L\left[10^{44} \mathrm{erg} \mathrm{s}^{-1}\right]\right)^{0.54} E(z)^{-0.93}$, this results in a mass of $M_{500}=5.25 \times 10^{13} \mathrm{M}_{\odot}$.

The central temperature is $2.92_{-0.87}^{+1.89} \mathrm{keV}$. The $M-T$ relation, $M_{500}=0.29 \times 10^{14} \mathrm{M}_{\odot}(T[\mathrm{keV}])^{1.62} E(z)^{-1.04}$, then predicts a mass of $M_{500}=1.62_{-0.72}^{+2.04} \times 10^{14} \mathrm{M}_{\odot}$. Unfortunately, the number of counts is not sufficient to assess the dynamical state of the cluster with the standard $\mathrm{X}$-ray proxies as we cannot determine reliable centroid shifts or power ratios. 


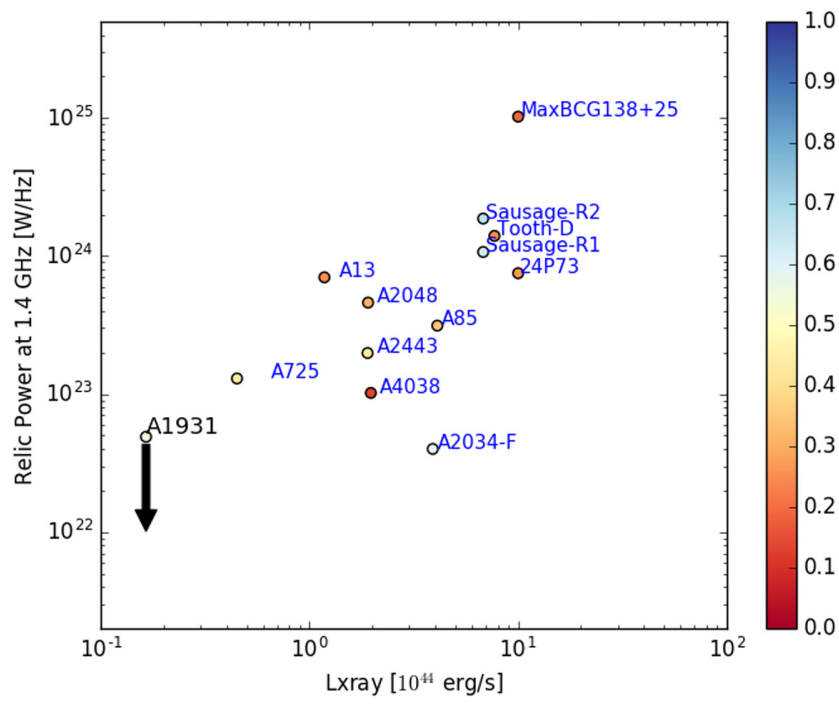

Figure 4. Specific luminosity at $1.4 \mathrm{GHz}$ of AGN/phoenix relics from Nuza et al. (2017) versus the X-ray luminosity of their host cluster in $10^{44} \mathrm{erg} \mathrm{s}^{-1}$. The colour of the points represents their largest linear scale/size in Mpc. The $\mathrm{X}$-ray luminosity of the clusters 24P73 and MaxBCG 138+25 is not known.

The cluster is not in the Planck cluster catalogue and is also not detected in the ROSAT All-Sky Survey (RASS). From the Planck non-detection, we conclude $M_{500}<3 \times 10^{14} \mathrm{M}_{\odot}$ (Planck Collaboration XIII 2016).

\section{DISCUSSION AND MODELLING}

The diffuse radio source sits close to the centre of the cluster but the morphology of the source and the fact that we do not have sufficient data at $325 \mathrm{MHz}$ to make a spectral index map does not allow an easy classification. The shape of the source makes it unlikely that it is a radio halo since it is neither round nor appears to fill the cluster centre.

Also a gischt-type radio relic appears unlikely. The steep spectrum is not consistent with a gischt relic that is associated with an active merger shock as those sources have integrated power-law spectra of $\sim-1$. Still, it is worth noting that the radio source appears to lie perpendicular to the merger axis of the cluster as suggested by the SDSS (see Fig. 1) and also the X-ray image (Fig. 3).

So far, most radio relics have been found pre-dominantly in massive galaxy clusters. In Fig. 4, we show the specific luminosity of AGN and phoenix-type radio relics at $1.4 \mathrm{GHz}$ versus the X-ray luminosity (with the largest linear size colour coded). These sources are taken from the recent list compiled in Nuza et al. (2017) and this list is clearly not complete. The upper limit for Abell 1931 is shown and it lies at the lower end of both X-ray and radio luminosities. In many ways, this source is similar to the diffuse radio source in the merging galaxy cluster Abell 2443 (also shown in Fig. 4) which also has a steep spectrum, is offset from the cluster centre, has an irregular morphology, and is not clearly associated with any of the galaxies within the cluster (Cohen \& Clarke 2011). This source is classified as a radio phoenix but the classification is uncertain.

Alternatively, the extended radio source in A1931 could be an AGN relic or remnant radio galaxy, similar to the one discovered by Brienza et al. (2017). Even though there is no detectable connection in the LOFAR image, the likely BCG of the cluster could be the origin of the remnant radio emission. Bulk motions along the
NE-SW directions could have moved the radio lobes from their source galaxy.

Customarily, the radio-synchrotron break frequency is used to estimate the age of the source. The break frequency $v_{\mathrm{b}}$ is related to the spectral age through

$t=1590 \frac{B^{1 / 2}}{B^{2}+B_{\mathrm{CMB}}^{2}}\left[v_{\mathrm{b}}(1+z)\right]^{-1 / 2} \mathrm{Myr}$,

where $B$ is given in $\mu \mathrm{G}$ and $v_{\mathrm{b}}$ in GHz. $B_{\mathrm{CMB}}$ is the magnetic field strength equivalent to the cosmic microwave background at the redshift of the source, $z$. Assuming the equipartition magnetic field of Beck \& Krause (2005) with a proton-to-electron ratio of 100 , we find $B_{\text {eq }}=1.9 \mu \mathrm{G}$ for $\alpha=-2.86$. However, this calculation assumes an extremely steep power law across the entire spectrum while in reality the spectrum may be curved. The injection spectrum at Lorentz factors where most of the energy density is contained is most likely to be flatter which would lead to a much weaker magnetic field. Also note that the associated magnetic pressure $B^{2} / 8 \pi \sim 1.4 \times 10^{-13} \mathrm{erg} \mathrm{cm}^{-3}$ is only a few per cent of the central pressure derived from the X-ray data which is $p \sim 8 \times 10^{-12} \mathrm{erg}$ $\mathrm{cm}^{-3}$.

At this field strength, the radiative losses are dominated by inverse Compton losses. We do not know what the break frequency is but it must be smaller than $\sim 140 \mathrm{MHz}$. Setting the break frequency to $140 \mathrm{MHz}$, we find that the spectral age must be larger than $\sim 220$ Myr.

Informed by hydrodynamical simulations of jet evolution, Hardcastle (2018) have modelled the evolution of remnant radio galaxies. They found that when the supply of fresh particles ceases, radiative and adiabatic losses lead to an almost instantaneous disappearance of the high-frequency emission. In this semi-analytic model, it is assumed that a constant bi-polar jet expands into a spherically symmetric and isothermal cluster that follows the so-called universal pressure profile for clusters (Arnaud 1996). A constant fraction (assumed to be 0.4 ) of the energy supplied by the light jet goes into the internal energy of the lobe. The jets drive a shock into the ICM that follows the non-relativistic Rankine-Hugoniot relations. The magnetic field energy density is assumed to be a constant fraction (0.1) of the electron energy density at all times. The lobes behave as an adiabatic fluid with an adiabatic index, $\Gamma=4 / 3$ while the external medium has $\Gamma=5 / 3$. The electrons are injected with a constant power-law index (in energy) of 2.1 and the maximum and minimum Lorentz factor assumed for the injected electrons are 10 and $10^{6}$, respectively. For more details on the model, we refer the reader to Hardcastle (2018).

Varying the energy of the jet, $Q$, as well as the time when the jet is turned off, $t_{\text {off }}$ as the only free parameters, we found that values of $t_{\text {off }}=50 \mathrm{Myr}$ and $Q=10^{36} \mathrm{~W}$ gave a lobe length of $R=200$ kpc, a specific luminosity of $P_{143 \mathrm{MHz}}=1.26 \times 10^{25} \mathrm{~W} \mathrm{~Hz}^{-1}$ and a spectral index between 143 and $325 \mathrm{MHz}$ of $\sim-2.8$ at a time of $\sim 190 \pm 20 \mathrm{Myr}$ where the errors are determined from the righthand panel in Fig. 5. A jet power of $10^{36} \mathrm{~W}$ is expected for AGN host galaxies with stellar masses between $10^{11}$ and $10^{12} \mathrm{M}_{\odot}$ (e.g. Shabala et al. 2008). Fig. 5 shows the radio luminosity as a function of time and a possible evolutionary path in the lobe length-spectral index $\alpha_{143}^{1500}$ space. For comparison, we have overlaid in transparent circles an evolutionary path for a model with $t_{\text {off }}=200 \mathrm{Myr}$, all other parameters staying the same. The width of the source with these model parameters is $\sim 50 \mathrm{kpc}$ which is also in line with the observations. This time-scale is in reasonable agreement with the estimate based on the synchrotron break frequency alone (which ignores adiabatic effects etc.). Clearly, we have only presented one 

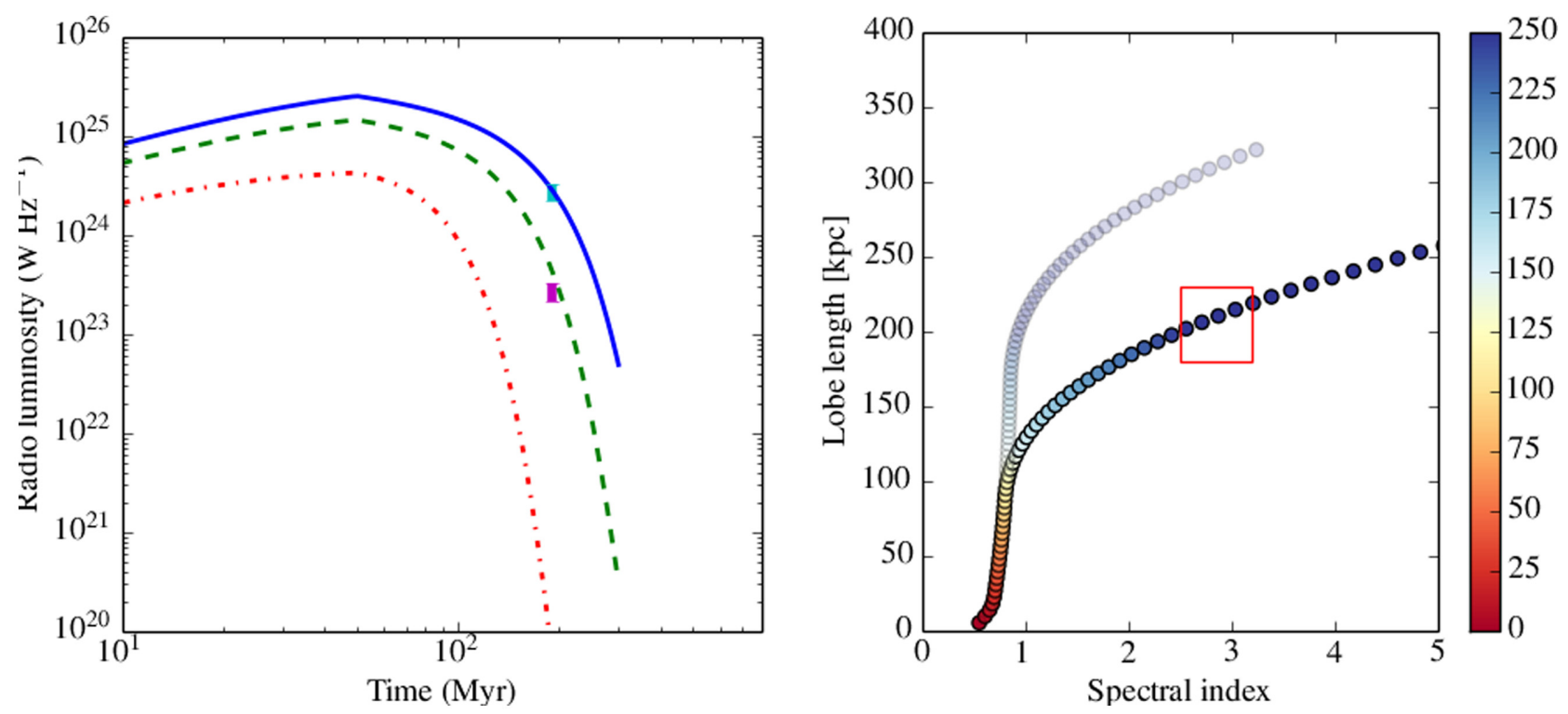

Figure 5. Left: Luminosity at $143 \mathrm{MHz}$ (solid blue), $325 \mathrm{MHz}$ (dashed green), and $1.5 \mathrm{GHz}$ (dotted red) as a function of time. Right: Length of lobe in kpc versus spectral index $\alpha_{143}^{325}$. Model assumes a universal pressure profile for a cluster of mass $M=10^{14} \mathrm{M}_{\odot}$, a duration of the active jet of $t_{\mathrm{off}}=50 \mathrm{Myr}$, a jet power of $Q=10^{36} \mathrm{~W}$, and a redshift of $z=0.178$. The errorbars and the red box, respectively, indicate measured values for A1931. Overlaid in transparent circles is an evolutionary path with all model parameters the same, only for $t_{\mathrm{off}}=200 \mathrm{Myr}$.

scenario within a somewhat idealized model and do not intend to perform an exhaustive modelling of this source given that the data is still very limited. The alleged centre of the diffuse radio source is located at a projected distance of about $100 \mathrm{kpc}$ from the presumed BCG. Bulk flows in the cluster could have advected the remnant galaxy over a distance of $100 \mathrm{kpc}$ within $120 \mathrm{Myr}$ assuming a velocity of $800 \mathrm{~km} \mathrm{~s}^{-1}$.

This rapid fading of the remnant sources has been invoked to explain the small number of remnant radio galaxies seen at low frequencies. Future searches are expected to reveal more about the effects and long-term evolution of radio jets in galaxy groups and clusters.

\section{SUMMARY}

We have discovered a diffuse, steep-spectrum radio source in the low-mass cluster Abell 1931. This source is only detected in LOFAR 120-168 MHz (HBA) and GMRT $325 \mathrm{MHZ}$ images. From the primary-beam-corrected image, we measure a total flux density for the source at $143 \mathrm{MHz}$ of $S_{143 \mathrm{MHz}}=39.7 \pm 8.0 \mathrm{mJy}$ yielding a power of $P_{143 \mathrm{MHz}}=2.78 \pm 0.56 \times 10^{24} \mathrm{~W} \mathrm{~Hz}^{-1}$. The source has a fairly irregular morphology with a largest linear size of about 550 $\mathrm{kpc}$. The optical image suggests that the cluster is in the midst of a merger.

We measure a total flux density for the diffuse emission from the GMRT image of $S_{325 \mathrm{MHz}}=3.85 \pm 0.82 \mathrm{mJy}$. The spectral index of the total emission between 143 and $325 \mathrm{MHz}$ is therefore $\alpha_{143}^{325}=-2.86 \pm 0.36$.

The diffuse radio source remained undetected in VLA $L$-band 1-2 GHz observations in $\mathrm{C}$ - and $\mathrm{B}$-configurations. This leads to an upper limit on the flux density of $S_{1-2 \mathrm{GHz}}<0.52 \mathrm{mJy}$, implying a spectral index between $143 \mathrm{MHz}$ and $1.5 \mathrm{GHz}$ of $\alpha_{143}^{1400}<-1.9$. Unlike the sources in Brienza et al. (2016) and Brienza et al. (2017) which are not in clusters, our source has a steep spectrum at low radio frequencies.
The cluster is not included in a Planck cluster catalogue and is also not detected in the ROSAT All Sky Survey. Dedicated Chandra $\mathrm{X}$-ray observations of the cluster revealed a bolometric luminosity of $L_{X}=(1.65 \pm 0.39) \times 10^{43} \mathrm{erg} \mathrm{s}^{-1}$ and a temperature of $2.92_{-0.87}^{+1.89}$ $\mathrm{keV}$ which implies a mass of $M_{500} \sim 10^{14} \mathrm{M}_{\odot}$.

Based on size, spectral slope, power and morphology, we conclude that this source is likely to be a remnant radio galaxy. Modelling using a semi-analytic model by Hardcastle (2018) suggest that the source has been produced by a source with a power of $Q=10^{36} \mathrm{~W}$ that was active for $t_{\mathrm{off}}=50 \mathrm{Myr}$ and is about $190 \mathrm{Myr}$ old. A bright, red elliptical galaxy (SDSS J143212.84+441620.4), possibly the BCG of the cluster, has a radio counterpart indicating it is currently active AGN. Hence, it is conceivable that it is one of the sources of the relativistic electrons that produce the synchrotron emission.

Thus, our observations afford a rare glimpse into faded sources whose role in feedback processes is not understood. Both the length of time between outbursts as well as the duration of radio outbursts are central to quantifying the importance of AGN feedback.

Finally, if this is indeed a merging galaxy cluster, it is interesting that there are no gischt-type relics or radio haloes present.

\section{ACKNOWLEDGEMENTS}

MB acknowledges support from the grant BR2026/24 from the Deutsche Forschungsgemeinschaft (DFG). AB acknowledges support from the ERC-StG 714245 DRANOEL. RJvW acknowledges support from the ERC Advanced Investigator programme NewClusters 321271 and the VIDI research programme with project number 639.042.729, which is financed by the Netherlands Organization for Scientific Research (NWO). LOFAR, the Low Frequency Array designed and constructed by ASTRON, has facilities in several countries, that are owned by various parties (each with their own funding sources), and that are collectively operated by the International LOFAR Telescope (ILT) foundation under a joint sci- 
entific policy. The LOFAR software and dedicated reduction packages on https://github.com/apmechev/GRID_LRT were deployed by the LOFAR e-infragroup, consisting of J.B.R. Oonk (ASTRON \& Leiden Observatory), A.P. Mechev (Leiden Observatory), and T. Shimwell (Leiden Observatory) with support from N. Danezi (SURFsara) and C. Schrijvers (SURFsara). This work has made use of the Dutch national e-infrastructure with the support of SURF Cooperative through grant e-infra160022. Support for this work was provided by the National Aeronautics and Space Administration through Chandra Award Number 19569 issued by the Chandra $\mathrm{X}$-ray Center, which is operated by the Smithsonian Astrophysical Observatory for and on behalf of the National Aeronautics Space Administration under contract NAS8-03060. This research has made use of software provided by the Chandra X-ray Center (CXC) in the application packages CIAO, CHIPS, and SHERPA. We thank the staff of the GMRT who have made these observations possible. GMRT is run by the National Centre for Radio Astrophysics of the Tata Institute of Fundamental Research. The NRAO is a facility of the National Science Foundation operated under cooperative agreement by Associated Universities, Inc. We acknowledge helpful conversations with Raffaella Morganti and parts of code written by Martin Hardcastle published under https://github.com/mhardcastle.

\section{REFERENCES}

Anders E. Grevesse N., 1989, Geochim. Cosmochim. Acta, 53, 197

Arnaud K. A., 1996, in Holt S. S., Sonneborn G., eds, ASP Conf. Ser. Vol. 99, Cosmic Abundances. Astron. Soc. Pac., San Francisco, p. 409

Beck R., Krause M., 2005, Astron. Nachr., 326, 414

Bonafede A., Intema H. T., Brüggen M., Girardi M., Nonino M., Kantharia N., van Weeren R. J., Röttgering H. J. A., 2014, ApJ, 785, 1

Brienza M. et al., 2016, A\&A, 585, A29

Brienza M. et al., 2017, A\&A, 606, A98

Briggs D. S., 1995, High Fidelity Deconvolution of Moderately Resolved Sources. New Mexico Institute of Mining Technology, Socorro, New Mexico, USA

Brunetti G., Jones T. W., 2014, Int. J. Mod. Phys. D, 23, 1430007

Brunetti G. et al., 2008, Nature, 455, 944

Cassano R., Brunetti G., Röttgering H. J. A., Brüggen M., 2010, A\&A, 509, A68

Cassano R. et al., 2013, ApJ, 777, 141

Cohen A. S., Clarke T. E., 2011, AJ, 141, 149

Cornwell T. J., Golap K., Bhatnagar S., 2005, in Shopbell P., Britton M., Ebert R., eds, ASP Conf. Ser. Vol. 347, Astronomical Data Analysis Software and Systems XIV. Astron. Soc. Pac., San Francisco, p. 86

Cornwell T. J., Golap K., Bhatnagar S., 2008, IEEE J. Sel. Top. Signal Process., 2, 647

Cuciti V., Cassano R., Brunetti G., Dallacasa D., Kale R., Ettori S., Venturi T., 2015, A\&A, 580, A97

de Gasperin F., van Weeren R. J., Brüggen M., Vazza F., Bonafede A., Intema H. T., 2014, MNRAS, 444, 3130

de Gasperin F. et al., 2017, Sci. Adv., 3, e1701634

Dennison B., 1980, ApJ, 239, L93

Dickey J. M. , Lockman F. J., 1990, ARA\&A, 28, 215

Dwarakanath K. S., Kale R., 2009, ApJ, 698, L163

Ensslin T. A., Biermann P. L., Klein U. Kohle S., 1998, A\&A, 332, 395

Enßlin T. A., Brüggen M., 2002, MNRAS, 331, 1011

Enßlin T. A., Gopal-Krishna , 2001, A\&A, 366, 26
Giovannini G. , Feretti L., Gregorini L. , Parma P., 1988, A\&A, 199, 73

Godfrey L. E. H., Morganti R., Brienza M., 2017, MNRAS, 471, 891

Greisen E. W., 2003, in Heck A., ed., Astrophysics and Space Science Library, Vol. 285, Information Handling in Astronomy - Historical Vistas. Kluwer Academic Publishers, Dordrecht, p. 109,

Guo X., Sironi L., Narayan R., 2014, ApJ, 797, 47

Hardcastle M. J., 2018, MNRAS, 475, 2768

Intema H. T., Jagannathan P., Mooley K. P., Frail D. A., 2017, A\&A, 598, A78

Kale R., Venturi T., Giacintucci S., Dallacasa D., Cassano R., Brunetti G., Macario G., Athreya R., 2013, A\&A, 557, A99

Kale R. et al., 2015, A\&A, 579, A92

Kang H., Ryu D., 2011, ApJ, 734, 18

Kang H., Ryu D., 2015, ApJ, 809, 186

Kempner J. C., Blanton E. L., Clarke T. E., Enßlin T. A., Johnston-Hollitt M., Rudnick L., 2004, in Reiprich T., Kempner J., Soker N., eds, The Riddle of Cooling Flows in Galaxies and Clusters of Galaxies, Available at: http://www.astro.virginia.edu/coolflow

Lane W. M., Cotton W. D., van Velzen S., Clarke T. E., Kassim N. E., Helmboldt J. F., Lazio T. J. W., Cohen A. S., 2014, MNRAS, 440, 327

Lopes P. A. A., de Carvalho R. R., Gal R. R., Djorgovski S. G., Odewahn S. C., Mahabal A. A., Brunner R. J., 2004, AJ, 128, 1017

McMullin J. P., Waters B., Schiebel D., Young W., Golap K., 2007, in Shaw R. A., Hill F., Bell D. J., eds, ASP Conf. Ser. Vol. 376, Astronomical Data Analysis Software and Systems XVI. Astron. Soc. Pac., San Francisc, p. 127

Mohan N., Rafferty D., 2015, Astrophysics Source Code Library, record ascl: 1502.007

Murgia M. et al., 2011, A\&A, 526, A148

Nuza S. E., Gelszinnis J., Hoeft M., Yepes G., 2017, MNRAS, 470, 240

Offringa A. R., de Bruyn A. G. , Biehl M. , Zaroubi S. , Bernardi G. , Pandey V. N., 2010, MNRAS, 405, 155

Parma P., Murgia M., de Ruiter H. R. , Fanti R., Mack K.-H. , Govoni F., 2007, A\&A, 470, 875

Pfrommer C. , Enßlin T. A., 2004, A\&A, 413, 17

Pinzke A., Oh S. P., Pfrommer C., 2013, MNRAS, 435, 1061

Planck Collaboration XIII, 2016, A\&A, 594, A13

Rau U., Cornwell T. J., 2011, A\&A, 532, A71

Reichert A., Böhringer H., Fassbender R., Mühlegger M., 2011, A\&A, 535, A4

Rengelink R. B., Tang Y., de Bruyn A. G., Miley G. K., Bremer M. N., Roettgering H. J. A., Bremer M. A. R., 1997, A\&AS, 124, 259

Savini F. et al., 2018, MNRAS, 474, 5023

Scaife A. M. M., Heald G. H., 2012, MNRAS, 423, L30

Shabala S. S., Ash S., Alexander P., Riley J. M., 2008, MNRAS, 388, 625

Shimwell T. W. et al., 2017, A\&A, 598, A104

van Haarlem M. P. et al., 2013, A\&A, 556, A2

van Weeren R. J. et al., 2016a, ApJS, 223, 2

van Weeren R. J. et al., 2016b, ApJ, 817, 98

van Weeren R. J. et al., 2017, Nat. Astron., 1, 0005

Vazza F., Brüggen M., Gheller C., Hackstein S., Wittor D., Hinz P. M., 2017, Class. Quantum Gravity, 34, 234001

Venturi T. Giacintucci S. Dallacasa D. Cassano R. Brunetti G. Bardelli S. Setti G., 2008, A\&A, 484, 327

Wen Z. L., Han J. L., 2013, MNRAS, 436, 275

Wilber A. et al., 2018, MNRAS, 473, 3536

This paper has been typeset from a $\mathrm{T}_{\mathrm{E}} \mathrm{X} / \mathrm{L} \mathrm{T} \mathrm{E} \mathrm{X}$ file prepared by the author. 